\title{
ON THE REPRESENTATION OF NONSEPARABLE ANALYTIC SETS
}

\author{
R. W. HANSELL
}

\begin{abstract}
Recently, the author considered extending the concepts of a Borel and analytic set for nonseparable metric spaces by allowing $\sigma$-discrete families to replace countable families in the classical definitions. The resulting class of "extended Borel sets" was shown to lead to a new class of sets, intermediate to the Borel and analytic sets. In the present paper we show that the corresponding class of "extended analytic sets" does not lead to a new class of sets but actually coincides with the standard class of analytic sets. Thus their importance lies in the fact that they provide a useful "representation" for the analytic sets in arbitrary spaces. Several such representations are shown to lead to the same class of analytic sets.
\end{abstract}

1. Introduction. It is well known that many of the deeper properties of Borel and analytic (also called Souslin) sets in the classical setting of a complete and separable metric space are no longer valid in the absence of separability. Such is the case for example with the celebrated Souslin Theorem (or, what amounts to the same thing, Lusin's First Separation Theorem [4, pp. 485-488]).

Recently, in [1] and [2] we investigated the possibility of extending the concepts of a Borel and analytic set for nonseparable metric spaces by allowing $\sigma$-discrete families to replace countable families in the classical definitions. This led to the fruitful notions of a $k$-Borel and $k$-Souslin set (where $k$ is an infinite cardinal), and a general theory of these sets which closely parallels the classical theory. In particular, generalizations of the two theorems mentioned above were obtained.

Now, it was shown in [2] that each $k$-Borel set of a space is an analytic set in the classical sense, but that the family of $k$-Borel sets was strictly larger than the family of Borel sets for most nonseparable spaces of infinite weight $k$. And a similar result was thought to be true also for the families of $k$-Souslin and Souslin (or analytic) sets.

Received by the editors September 19, 1972.

AMS (MOS) subject classifications (1970). Primary 54H05, 04A15; Secondary 54C10, 54E35.

Key words and phrases. Analytic set, Borel set, $k$-Souslin representations.

(c) American Mathematical Society 1973 
Unexpectedly however, it turns out (as we will presently show) that rather than constituting an extension of the family of analytic sets, the $k$-Souslin sets are actually a different way of representing these classical sets in nonseparable metric spaces. Thus, rather than viewing the theory of $k$-Borel and $k$-Souslin sets as a generalization of the classical theory, what we have really found is a new family of "extended Borel" sets ${ }^{1}$ which are intermediate to the Borel and analytic sets; and it is to these extended Borel sets that the nonseparable analytic sets relate in a natural way and which leads to a natural generalization of the classical separable theory.

2. Definitions and preliminaries. An indexed family $\left\{A_{t} \mid t \in T\right\}$ of subsets of a space $X$ is said to be discrete in $X$ if each point of $X$ has a neighborhood $U$ such that $U \cap A_{t} \neq \varnothing$ for at most one $t \in T$. It is said to be $\sigma$ discrete if we can write $T=\bigcup_{n=1}^{\infty} T_{n}$ such that $\left\{A_{t} \mid t \in T_{n}\right\}$ is discrete for each $n$. On the other hand, the family $\left\{A_{t} \mid t \in T\right\}$ is said to be $\sigma$-discretely decomposable ( $\sigma$-d.d.) if there exist sets $A_{t n}(t \in T, n=1,2, \cdots)$ such that $\left\{A_{t n} \mid t \in T\right\}$ is discrete for fixed $n$ and each $A_{t}=\bigcup_{n=1}^{\infty} A_{t n}$.

Now, for each infinite cardinal $k$, let $T_{n}(k)$ be a given set of cardinality $k$ (for $n=1,2, \cdots$ ) and set $B(k)=\prod T_{n}(k)$. For $t \in B(k)$ we denote the $i$ th coordinate of $t$ by $t_{i}$ and the $n$-tuple $\left(t_{1}, \cdots, t_{n}\right)$ by $t(n)$. The set $\{t(n) \mid t \in B(k)\}$ will be denoted by $B_{n}(k)$.

2.1. Definition. A subset $S$ of a topological space $X$ is said to be a $k$-Souslin set in $X$ if there exist closed subsets $F_{t(n)}$ of $X$, for $t(n) \in B_{n}(k)$ and $n=1,2, \cdots$, such that

$$
F\left(1_{k}\right) \quad S=\bigcup_{t \in B(k)} \bigcap_{n=1}^{\infty} F_{t(n)},
$$

and

$F\left(2_{k}\right) \quad\left\{F_{t(n)} \mid t(n) \in B_{n}(k)\right\}$ is $\sigma$-discrete for each $n$.

It is immediate that the classical analytic sets [5], [6] (or Souslin sets in the terminology of [3]) coincide with the $\boldsymbol{K}_{0}$-Souslin sets. Note that sets which have property $F\left(1_{k}\right)$ are called $k$-analytic in [7].

In [2] we showed that property $F\left(2_{k}\right)$ could be weakened by replacing the word " $\sigma$-discrete" by " $\sigma$-d.d.". More generally, we proved the following which we will need here (see [2, Theorem 4.2]):

2.2. If

$$
\left\{E_{t(n)} \mid t(n) \in B_{n}(k), n=1,2, \cdots\right\}
$$

\footnotetext{
${ }^{1}$ By the extended Borel sets of a metric space $X$ we mean the smallest family of subsets of $X$, containing the open sets, and closed with respect to complementation and unions of $\sigma$-discrete families (see [2]).
} 
is a system of subsets of a topological space $^{2} X$ such that each family obtained by fixing $n$ is $\sigma$-d.d., and if

$$
\left.S=\bigcup_{t \in B(k)} \bigcap_{n=1}^{\infty} E_{t(n)}=\bigcup_{t \in B(k)} \bigcap_{n=1}^{\infty} \bar{E}_{t(n)} \quad \text { (closure in } X\right),
$$

then $S$ is a $k$-Souslin set in $X$.

An equivalent but somewhat less natural definition of a $k$-Souslin set was given in [1]: A set $S$ is $k$-Souslin in $X$ if there exist (not necessarily closed) sets $S_{t(n)} \subset X$ satisfying

$S\left(1_{k}\right) \quad S=\bigcup\left\{S_{t(1)} \mid t \in B(k)\right\}$,

$S\left(2_{k}\right) \quad S_{t(n)}=\bigcup\left\{S_{s(n+1)} \mid s \in B(k)\right.$ and $\left.s(n)=t(n)\right\}$,

$S\left(3_{k}\right) \quad\left\{S_{t(n)} \mid t(n) \in B_{n}(k)\right\}$ has a $\sigma$-discrete base for each $n,^{3}$

and

$S\left(4_{k}\right)$

$$
\bigcap_{n=1}^{\infty} S_{t(n)}=\bigcap_{n=1}^{\infty} S_{t(n)} \text { for each } t \in B(k) .
$$

Part of our main theorem is to show that the two definitions are indeed equivalent.

\section{The main theorem.}

3.1. THEOREM. For any subset $S$ of a topological space $X$ and any infinite cardinal $k$ the following are equivalent:

(1) $S$ is an analytic set in $X$.

(2) $S$ is a $k$-Souslin set in $X$.

(3) $S$ can be represented by a system $\left\{S_{t(n)} \mid t(n) \in B_{n}(k), n=1,2, \cdots\right\}$ of subsets of $X$ satisfying properties $S\left(1_{k}\right)-S\left(4_{k}\right)$.

Proof. (1) implies (2). Suppose $S=\bigcup_{t \in B\left(\aleph_{0}\right)} \bigcap_{n=1}^{\infty} F_{t(n)}$ where each $F_{t(n)}$ is a closed subset in $X$. Since $k \geqq \aleph_{0}$ we may assume that the indexing sets are such that $T_{n}(k) \supset T_{n}\left(\boldsymbol{\aleph}_{0}\right)$ for each $n$. Hence we can define

$$
\begin{aligned}
F_{t(n)}^{*} & =F_{t(n)} & & \text { if } t(n) \in B_{n}\left(\boldsymbol{\aleph}_{0}\right), \\
& =\varnothing & & \text { if } t(n) \in B_{n}(k)-B_{n}\left(\boldsymbol{\aleph}_{0}\right),
\end{aligned}
$$

${ }^{2}$ Strictly speaking, all the theorems of [2] were stated for the case when $X$ is metrizable, since the main results depended upon this. However, it is easy to verify that most of the preliminary results of [2] (including Theorem 4.2) use only the fact that if a family of sets is discrete, then the family of their closures is discrete and the closure of the union is the union of the closures, which is true in any topological space. I thank the referee for kindly pointing out the fact that the proof of the main theorem of this note is true for arbitrary topological spaces.

${ }^{3} \mathrm{~A}$ family $\mathscr{B}$ of sets is said to be a base for a family $\mathscr{A}$ if each member of $\mathscr{A}$ is a union of members from $\mathscr{B} ;$ a $\sigma$-discrete base is a base which is also $\sigma$-discrete. 
and one easily shows that the system $\left\{F_{t(n)}^{*}\right\}$ is a $k$-Souslin representation for $S$ in $X$.

(2) implies (3). Let $\left\{F_{t(n)}\right\}$ be a $k$-Souslin representation for $S$ in $X$ satisfying $F\left(1_{k}\right)$ and $F\left(2_{k}\right)$. For each $t(n) \in B_{n}(k)$ define

$$
S_{t(n)}=\bigcup_{s} \bigcap_{m=1}^{\infty} F_{s(m)}
$$

where the union is over all $s \in B(k)$ with $s(n)=t(n)$. The verification of properties $S\left(1_{k}\right)$ and $S\left(2_{k}\right)$ is routine (cf. [3, p. 205]). Since by (4) $S_{t(n)} \subset F_{t(n)}$ for each $t(n)$, it follows from $F\left(2_{k}\right)$ that $\left\{S_{t(n)}\right\}$ is also $\sigma$-discrete for fixed $n$, and thus $S\left(3_{k}\right)$ is satisfied. Also, $S_{t(n)} \subset F_{t(n)}$ implies that $\bigcap_{n=1}^{\infty} S_{t(n)} \subset$ $\bigcap_{n=1}^{\infty} F_{t(n)}$. On the other hand, we have $\bigcap_{n=1}^{\infty} F_{t(n)} \subset S_{t(m)}$ for each $m=$ $1,2, \cdots$ by (4), and so $\bigcap_{n=1}^{\infty} F_{t(n)} \subset \bigcap_{n=1}^{\infty} S_{t(n)}$. Property $S\left(4_{k}\right)$ is thus satisfied.

We now show that (3) implies (2) and then, finally, that (2) implies (1).

Assuming (3), for each $n$ let $\mathscr{B}_{n}=\bigcup_{n=1}^{\infty} \mathscr{B}_{n m}$ be a $\sigma$-discrete base for $\left\{S_{t(n)} \mid t(n) \in B_{n}(k)\right\}$, where each $\mathscr{B}_{n m}$ is discrete. We may assume that each of the indexing sets $T_{n}(k)$ is well-ordered by a fixed relation, and that the set $B_{n}(k)$ is then well-ordered lexicographically for each $n$ (i.e., $s(n)<t(n)$ iff $s(1)<t(1)$ or, for some $i=1, \cdots, n-1, s(i)=t(i)$ and $\left.s_{i+1}<t_{i+1}\right)$.

Now define the collections

$$
\mathscr{B}_{n m}^{t(n)}=\left\{B \in \mathscr{B}_{n m} \mid t(n) \text { is the least index in } B_{n}(k) \text { such that } B \subset S_{t(n)}\right\} \text {, }
$$
and put

$$
E_{t(n)}^{m}=\bigcup \mathscr{B}_{n m}^{t(n)} \quad \text { and } \quad E_{t(n)}=\bigcup_{m=1}^{\infty} E_{t(n)}^{m}
$$

Since, by (5), each nonempty $B \in \mathscr{B}_{n m}$ belongs to at most one $\mathscr{B}_{n m}^{t(n)}$ for fixed $n$ and $m$, it follows that the family $\left\{E_{t(n)}^{m} \mid t(n) \in B_{n}(k)\right\}$ is discrete, and hence that $\left\{E_{t(n)} \mid t(n) \in B_{n}(k)\right\}$ is $\sigma$-discretely decomposable. Thus, to show that $S$ is a $k$-Souslin set in $X$, it suffices by 2.2 to show that

$$
S=\bigcup_{t \in B(k)} \bigcap_{n=1}^{\infty} E_{t(n)}=\bigcup_{t \in B(k)} \bigcap_{n=1}^{\infty} \bar{E}_{t(n)} .
$$

First note that by (5) and (6) we have $E_{t(n)}^{m} \subset S_{t(n)}$ for each $m$, and so $E_{t(n)} \subset S_{t(n)}$. Hence it follows that

$$
\bigcap_{n=1}^{\infty} \bar{E}_{t(n)} \subset \bigcap_{n=1}^{\infty} \bar{S}_{t(n)}=\bigcap_{n=1}^{\infty} S_{t(n)} \subset S
$$

for each $t \in B(k)$ (where we have used $S\left(4_{k}\right)$ ). 
Now suppose $x \in S$. We show there exists a point $s$ in $B(k)$ such that $s(n)$ is the least index in $B_{n}(k)$ with $x \in S_{s(n)}$. Defining the coordinates of $s$ inductively, by $S\left(1_{k}\right)$ let $S_{1}$ be the least index in $T_{1}(k)$ such that $x \in S_{s_{1}}$. Since by $S\left(2_{k}\right)$ the set of indices $t_{2}$ in $T_{2}(k)$ such that $x \in S_{s_{1} t_{2}}$ is not empty, we can choose a least one, $s_{2}$ say. Repeating this argument for the set $\left\{t_{3} \in T_{3}(k) \mid x \in S_{s_{1} s_{2} t_{3}}\right\}$, we obtain the coordinate $s_{3}$, etc. This defines a point $s$ in $B(k)$.

Now suppose some $s(n)$ is not the least member of $B_{n}(k)$ such that $x \in S_{s(n)}$, and let $m$ denote the smallest value of $n$ for which this is the case; i.e., there exists some $t(m)<s(m)$ such that $x \in S_{t(m)}$. Evidently $m>1$ by our definition of $s_{1}$, and so for some $i=2, \cdots, m-1$ we must have $t(i)=s(i)$ and $t_{i+1}<s_{i+1}$. But then

$$
x \in S_{t(m)} \subset S_{t(i+1)}=S_{s_{1} \cdots s_{i} t_{i+1}}
$$

and this contradicts the definition of $s_{i+1}$. Hence our assumption must be false, and it follows that $s$ has the desired properties.

Now, since $\mathscr{B}_{n}$ is a base for $\left\{S_{t(n)} \mid t(n) \in B_{n}(k)\right\}$, there exist indices $m_{n}$ and corresponding sets $B_{n} \in \mathscr{B}_{n m_{n}}$, for $n=1,2, \cdots$, such that $x \in B_{n} \subset$ $S_{s(n)}$. By the properties of $s$, this implies that $B_{n} \in \mathscr{B}_{n m_{n}}^{s(n)}$ and hence that $x \in E_{s(n)}^{m_{n}}$ by (5) and (6). It follows that $x \in E_{s(n)}$, and we have shown that

$$
S \subset \bigcup_{t \in B(k)} \bigcap_{n=1}^{\infty} E_{t(n)} .
$$

Since (7) follows from (8) and (9), we now have that $S$ is a $k$-Souslin set in $X$.

Finally, to prove that (2) implies (1) let $S \subset X$ be a $k$-Souslin set in $X$ with representation $\left\{F_{t(m)} \mid t(m) \in B_{m}(k), m=1,2, \cdots\right\}$ satisfying $F\left(1_{k}\right)$ and $F\left(2_{k}\right)$. Accordingly, for each $m$, we assume that

$$
\left\{F_{t(m)} \mid t(m) \in B_{m}(k)\right\}=\bigcup_{n=1}^{\infty} \mathscr{F}_{m n}
$$

where each $\mathscr{F}_{m n}$ is discrete in $X$, and that $F_{t(m+1)} \subset F_{t(m)}$ for each $t \in B(k)$. (The latter can always be done; cf. $[4, \S 3$, p. 31$]$.)

Now define the families

$$
\mathscr{A}_{n_{1} \cdots n_{\nu}}=\left\{F_{t(p)} \mid F_{t(m)} \in \mathscr{F}_{m n_{m}} \text { for } m=1, \cdots, p\right\},
$$

for each $p$-tuple $\left(n_{1}, \cdots, n_{p}\right)$ of natural numbers, $p=1,2, \cdots$, and put

$$
A_{n_{1} \cdots n_{p}}=\bigcup \mathscr{A}_{n_{1} \cdots n_{p}} \text {. }
$$

Since $\mathscr{A}_{n_{1} \cdots n_{p}} \subset \mathscr{F}_{p_{n_{p}}}$, it is discrete, and so each $A_{n_{1} \cdots n_{p}}$ is a closed subset 
of $X$. Hence we need only show that

$$
S=\bigcup_{\left(n_{p}\right)} \bigcap_{p=1}^{\infty} A_{n_{1} \cdots n_{p}},
$$

where the union is over all $\left(n_{p}\right) \in B\left(\aleph_{0}\right)$, to prove that $S$ is analytic in $X$.

Thus suppose $x \in S$, say $x \in \bigcap_{p=1}^{\infty} F_{t(p)}$. Since for each $p=1,2, \cdots$, there exists an index $n_{p}$ such that $F_{t(p)} \in \mathscr{F}_{p n_{p}}$, it follows by (10) and (11) that $x \in \bigcap_{p=1}^{\infty} A_{n_{1} \cdots n_{p}}$. On the other hand, suppose $x \in \bigcap_{p=1}^{\infty} A_{n_{1} \cdots n_{p}}$ for some $\left(n_{1}, n_{2}, \cdots\right) \in B\left(\aleph_{0}\right)$. Then $x$ belongs to some $F_{s_{1}} \in \mathscr{A}_{n_{1}}=\mathscr{F}_{1_{n_{1}}}$. Proceeding inductively, assume we have defined coordinates $s_{m}$ such that $x \in F_{s_{1} \cdots s_{m}} \in \mathscr{F}_{m n_{m}}$ for $m=1, \cdots, p-1$. Since $x \in A_{n_{1} \cdots n_{p}}, x$ belongs to some $F_{t(p)} \in \mathscr{F}_{p n_{p}}$ with $F_{t(m)} \in \mathscr{F}_{m n_{m}}$ for $m=1, \cdots, p-1$. But then $x$ is a member of both $F_{s(p-1)}$ and $F_{t(p-1)}$ and both these sets belong to the discrete family $\mathscr{F}_{p-1, n_{p-1}}$. Consequently, we must have $s(p-1)=t(p-1)$ and hence we need only define $s_{p}=t_{p}$ to complete the induction.

It follows that $x \in \bigcap_{p=1}^{\infty} F_{s(p)} \subset S$, and the equality in (12) is established.

This completes the proof of the theorem.

REMARK. It was shown in $[2, \S 4.1]$ that property $F\left(2_{k}\right)$ in the definition of a $k$-Souslin set cannot be weakened by replacing the words "is $\sigma$ discrete" by the words "has a $\sigma$-discrete base." We now give an example to show that property $S\left(3_{k}\right)$ cannot be weakened to

$S\left(3_{k}\right)^{\prime} \quad\left\{S_{t(n)} \mid t(n) \in B_{n}(k)\right\}$ has a $\sigma$-discrete refinement for each $n$

in the alternate definition.

Let $S$ be any infinite set of real numbers, say $|S|=k$. We will show that $S$ has a representation $\left\{S_{t(n)}\right\}$ satisfying $S\left(1_{k}\right), S\left(2_{k}\right), S\left(4_{k}\right)$ and $S\left(3_{k}\right)^{\prime}$. For let $s_{1}, s_{2}, \cdots$ be any sequence of distinct points in $S$ and define for each $n$ tuple $\left(t_{1}, \cdots, t_{n}\right)$ in $S^{n}=S \times \cdots \times S$ ( $n$ factors)

$$
\begin{aligned}
S_{t(n)} & =\left\{t_{1}\right\} & & \text { if } t_{1} \notin\left\{s_{1}, s_{2}, \cdots\right\}, \\
& =\left\{t_{i+1}\right\} & & \text { if } t_{1}=s_{i} \text { for some } i=1, \cdots, n-1, \\
& =S & & \text { if } t_{1} \in\left\{s_{n}, s_{n+1}, \cdots\right\},
\end{aligned}
$$

(with the convention that when $n=1$ the second does not apply). Properties $S\left(1_{k}\right), S\left(2_{k}\right)$ and $S\left(4_{k}\right)$ are routine to verify. Finally, $S\left(3_{k}\right)^{\prime}$ holds since $\{S\}$ is a $\sigma$-discrete refinement for $\left\{S_{t(n)} \mid t(n) \in S^{n}\right\}$ for each $n$. Since there are nonanalytic subsets of real numbers, it follows from the equivalence of (1) and (3) of Theorem 3.1 that $S\left(3_{k}\right)$ cannot be weakened to $S\left(3_{k}\right)^{\prime}$.

\section{REFERENCES}

1. R. W. Hansell, On the nonseparable theory of Borel and Souslin sets, Bull. Amer. Math. Soc. 78 (1972), 236-241. 
2. R. W. Hansell, On the nonseparable theory of $k$-Borel and $k$-Souslin sets, General Topology and Appl. (to appear).

3. F. Hausdorff, Mengenlehre, 3rd ed., de Gruyter, Berlin, 1937; English transl., Chelsea, New York, 1957. MR 19, 111.

4. K. Kuratowski, Topologie. Vol. 1, PWN, Warsaw, 1958; English transl., Academic Press, New York; PWN, Warsaw, 1966. MR 19, 873; MR 36 \#840.

5. N. Lusin, Les ensembles analytiques, Paris, 1930.

6. W. Sierpiński, General topology, 2nd. ed., Univ. of Toronto Press, Toronto, 1952.

7. A. H. Stone, Non-separable Borel sets, Rozprawy Mat. 28 (1962). MR 27 \#2435.

Departivent of Mathematics, University of Connecticut, Storrs, Connecticut 06268 